\title{
Electronically Controlled Plasma Power Devices for Sustainable and Environmentally Friendly Electric Energy Technologies
}

\author{
M.V Dolgopolov ${ }^{1 *}$, V.V Radenko, G.G Zanin ${ }^{1}$, D.E Ovchinnikov ${ }^{1}$, A.V Radenko', \\ V.B Svirkov ${ }^{1}$, A.S Chipura ${ }^{2}$, A.V Gurskaya ${ }^{3}$ \\ ${ }^{1}$ New Energy RSC LLC, Zavodskoye sh. 25/2 Samara 443022, Russia \\ ${ }^{2}$ Samara University, Moskovskoye sh. 34 Samara 443086, Russia \\ ${ }^{3}$ Samara Polytech, Molodogvardeyskaya str. 244 Samara 443100, Russia \\ *Corresponding author. Email: mikhaildolgopolov68@gmail.com
}

\begin{abstract}
The experimental electronically controlled plasma power system is presented, with an estimated heat output ranging from 8 to $50 \mathrm{~kW}$, and an electrical output ranging from 5 to $35 \mathrm{~kW}$. The installation is based on the physical principles of ion and plasma fluxes compaction with discrete changes in the control parameters of magneto-optical systems. Authors use the latest achievements of materials science, microelectronics and energy industry to create sustainable generators for environmentally friendly green energy production technologies. The purpose of the project is an optimization of the operation of magneto-optical control systems of the plasma electric generator and a modification of the electronically controlled plasma electric generator from 5 to $50 \mathrm{~kW}$ to solve the problem of obtaining an energy source of compact size and mobile design that produces electrical energy and heat.
\end{abstract}

\section{Keywords: Electric Energy, Electronically Controlled Plasma, Magneto-optical control}

\section{INTRODUCTION}

This project is based on an experimental electronically controlled plasma power device manufactured by the team of authors, experimental estimates of the thermal power of which are in the range from 8 to $25 \mathrm{~kW}$, electric power-from 4 to $12.5 \mathrm{~kW}$. The operation of the installation is based on the physical principles of compaction of plasma and ion flows with a discrete change in the control parameters of magneto-optical systems.

The main objectives of this project are to study its operation, optimize output characteristics, modernize subsystems in order to create a serial sample with the useful output power of up to $50 \mathrm{~kW}$ as a source of electric energy for commercialization and industrial production.

In general, this topic is quite widely known, although the solution to its problems has not yet been determined. It relates, in general, to plasma physics and, in particular, concerns a device that allows for thermonuclear fusion, and for converting the energy of nuclear fusion products into electricity.

Thermonuclear fusion is the process by which two light nuclei combine to form a heavier core. During the thermonuclear process, a huge amount of energy is released in the form of fast-moving particles. Since atomic nuclei are positively charged due to the protons contained in them, an electrostatic or Coulomb repulsive force arises between them. In order for two nuclei to enter into a thermonuclear reaction, it is necessary to overcome this barrier of repulsion, which happens if two nuclei are brought close enough to each other, where the shortrange nuclear forces become sufficient to overcome the Coulomb force and start the process of thermonuclear fusion of these nuclei. The energy required for the nuclei to overcome the Coulomb barrier is provided by their thermal energy, which must be very high. For example, the rate of a thermonuclear reaction can become significant 
if the temperature is at least on the order of $104 \mathrm{eV}$, which approximately corresponds to 100 million degrees Kelvin. The rate of a thermonuclear reaction is a function of temperature and is characterized by a value called reactivity. The reactivity of the D-T reaction, for example, has a wide peak between $30 \mathrm{keV}$ and $100 \mathrm{keV}$.

The purpose of this work in the above-mentioned perspective is to obtain electronically controlled ionplasma flows for the initialization of thermonuclear fusion, followed by the retention of electronically controlled thermonuclear fusion in linear magnetic traps.

There are no analogues of this development. But attempts to develop such a system in practice have appeared. The device described in the patent [1] can be accepted as the some analogue of the proposed invention. The patent discloses a device for controlled thermonuclear fusion in a magnetic topology with a reversed field configuration (FRC) and converting the energy of thermonuclear fusion products directly into electrical energy. Plasma ions are preferably held by a magnetic field in the FRC configuration, while plasma electrons are held by an electrostatic field in a deep potential well created by adjusting an externally applied magnetic field. In this configuration, ions and electrons can have the required density and temperature, so that during collisions they enter into a thermonuclear reaction under the action of nuclear forces, thereby forming thermonuclear fusion products that arise in the form of a ring beam. The energy is taken from the ions of the fusion products as they move in a spiral past the electrodes of the reverse cyclotron converter. The advantage is that plasma flows from thermonuclear fuel, which can be used with a real energy retention and conversion system, include advanced (neutron-free) fuels.

In comparison with above mentioned, the advantages of our device are its functional simplicity and advanced features: modularity and compactness of execution, configurability of the system, the possibility of combining individual generators in a synchronously operating monoblock, control of the generator at the hardware and software level. Other types of reactions are also possible, for example, to produce neutrons.

\section{METHODS}

The report presents an electronically controlled plasma generator. The main goal is to solve the problem of electricity and heat supply independently of the centralized network, both in the mode of a backup energy source and the main power source.

Highlights of our installation system works according to the following principles:

1) acceleration of ions (also a separate controlled output of electrons),
2) variable compression of successive discrete ion fluxes by a magnetic lens,

3) ensuring quasi-stability by converting "outgoing fluxes" into rotation,

4) braking of the frontal current, which becomes the target, and collision with new particle fluxes (in fact, synthesis on oncoming beams),

5) ensuring "multi-passability" of ion currents through the active zone chamber,

6) external electrical synchronization of the system, due to which point 5) above is also provided.

The operation of an electronically controlled plasma electric generator PPS - XX V1.0 (Power Plant ECEG, Plasma Power System) is based on the reaction of nuclear fusion in ion flows [2-5] obtained as the result of evaporation of lithium hydride in the cartridge-evaporator, which is the cylindrical container with a tungsten or tungsten-rhenium heater, and ionization of the resulting vapor in an ionizer, which is a three-grid vacuum device that provides ionization of hydrogen and lithium vapors and their output to the section of a magneto-optical ion storage device, which is the magneto-optical trap, structurally consisting of a combination of solenoidal and quadrupole magnetic systems, and the subsequent acceleration of ions, setting a certain law of discrete change in the parameters of the magnetic control system and the energy distribution of the generated ion fluxes $[6,7]$ with the given parameters of nuclear fusion, implemented in a pulsed accelerator, which is a flight accelerating tube with input and output electrodes, and a magnetic sweep of the flow at the end of the accelerator. Nuclear fusion occurs in a magneto-optical n-cycle fusion chamber, which is designed as a combination of sequential solenoidal-quadrupole magneto-optical systems.

Energy generation is carried out by the electronically controlled plasma electric generator from the generated controlled ion or plasma flows in a multi-cycle synthesis chamber, and due to the subsequent conversion of the energy of the synthesis products into electrical energy - in the quantum energy converter (QEC), which is the ion-emission electrovacuum device (QEC1 ), which is structurally made in the form of a high-frequency triode with an input cylindrical electrode acting as a cathode, and a modulating grid placed at a short distance from the cathode, and a multi-grid anode with an external cylindrical coil creating a magnetic field inside the anode,operating on high-energy ions at a frequency of $50 \mathrm{kHz}$ and higher, or converting into an electric energy in the overhead electric vacuum klystron (QEC-2), which is structurally made in the form of the span klystron with an input resonator and four output resonators connected in parallel,operating on high- 
Table 1. Fusion reactions supported in the considered generator

\begin{tabular}{|l|c|c|c|}
\hline \multicolumn{1}{|c|}{ Reaction } & Energy release, in MeV & $\begin{array}{c}\text { omax in the energy range of } \\
1 \mathrm{MeV}\end{array}$ & $\begin{array}{c}\text { Energy of the incident particle } \\
\text { omax, Mev }\end{array}$ \\
\hline $1 \mathrm{p}+6 \mathrm{Li} \rightarrow 4 \mathrm{He}+3 \mathrm{He}$ & 4,0 & $10^{-4}$ & 0,3 \\
\hline $2 \mathrm{p}+7 \mathrm{Li} \rightarrow 24 \mathrm{He}+\mathrm{Y}$ & 17,3 & $6 \times 10^{-3}$ & 0,25 \\
\hline $3 \mathrm{p}+9 \mathrm{Be} \rightarrow 24 \mathrm{He}+\mathrm{d}$ & 0,56 & 0,46 & 0,33 \\
\hline $4 \mathrm{p}+9 \mathrm{Be} \rightarrow 6 \mathrm{Li}+4 \mathrm{He}$ & 2,1 & 0,35 & 0,33 \\
\hline $5 \mathrm{p}+11 \mathrm{~B} \rightarrow 34 \mathrm{He}$ & 8,7 & 0,6 & 0,675 \\
\hline $6 \mathrm{p}+15 \mathrm{~N} \rightarrow 12 \mathrm{C}+4 \mathrm{He}$ & 5,0 & $0,69($ at $1.2 \mathrm{MeV})$ & 1,2 \\
\hline
\end{tabular}

energy ions at a frequency of $2500 \mathrm{MHz}$ and higher. The substantiation of the scientific, technical and technological novelty of the developed scientific and technical solutions underlying the created product is given. Some similar developments were considered also in [810].

The device is realized by an ion accelerator with elements of high-current electronics, forming in a complex an electronically controlled pulse system, illustrated by the following schematic assembly drawing in Figure 1. An electronically controlled plasma electric generator structurally consists of: 1 - a lithium hydride cartridge; 2 -ion-plasma source; 3 - section of the magnetooptical storage device; 4 - accelerating section; 5 magneto-optical 4 (or 8) -cycle synthesis chamber; 6 transit high-frequency triode - ion-emission quantum energy converter (QEC); 7 - ionic vacuum pump neutralizer.

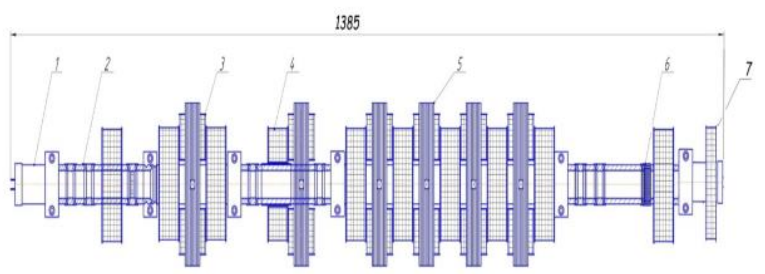

Figure 1. General assembly drawing of an electronically controlled plasma electric generator.

\subsection{Brief description of the criteria for evaluat- ing processes in the generator}

It is well known, that plasma is determined at temperatures above $10^{8} \mathrm{~K}$ and the Lawson test $\left(n \tau>10^{14} / \mathrm{m}^{3}\right.$ $\mathrm{s}$, where $n$ is the density of the high-temperature plasma, $\tau$ is the time of its retention in the system). The minimal energy of the incident flux must be no less than the threshold energy [9] $0.25 \mathrm{MeV}$ for the reaction $p+{ }^{7} \mathrm{Li} \rightarrow$ $2^{4} \mathrm{He}+\gamma$ at the energy yield of $17.3 \mathrm{MeV}$.

The possible types of nuclear reactions supported in the considered electronically controlled plasma generator are indicated in the Table 1.

In our case, the energy of the target and the energy of the incident flow are equal. The energy required for fusion is determined by the energy expended to accelerate ions and protons. The total energy expended consists of the energy for the vaporization of lithium hydride, the ionization energy of lithium and hydrogen, the acceleration of ions and protons, and the energy spent on magnetic systems. In our case:

- Temperature above $3.017 \times 10^{9} \mathrm{~K}$ at ion energy $E=260$ $\mathrm{keV}$

- The target density in the synthesis chamber $n=2.785 \mathrm{x}$ $10^{26} \mathrm{sm}^{-3}$

$=$ The retention time in the chamber is not less than $t=0.75 \mathrm{~s}$ (three flows in $0.25 \mathrm{~s}$ ) Lawson's criterion $n \tau>$ $2.088 \times 10^{26} \mathrm{~s} / \mathrm{sm}^{3}$.

The calculation of the generator energy was carried out with different amounts of the substance involved in the synthesis. Setting the number of ions NLi, $\mathrm{NH}$ and taking $\mathrm{NLi}=\mathrm{NH}$, we find the energy re-quired

Table 2. Estimates of the generator power for different amounts of the substance involved in the synthesis

\begin{tabular}{|l|c|c|c|c|c|}
\hline $\begin{array}{c}\text { Number of } \\
\mathrm{LiH} \text { moles }\end{array}$ & $\begin{array}{c}\text { Number of } \\
\text { atoms }\end{array}$ & $\begin{array}{c}\text { Ions current, } \\
\mathrm{A}\end{array}$ & $\begin{array}{c}\text { Mass LiH, } \\
\mathrm{kg}\end{array}$ & $\begin{array}{c}\text { Energy expended for } \\
\text { Synthesis, Esin, J }\end{array}$ & $\begin{array}{c}\text { Energy from fusion } \\
\text { Eout, J }\end{array}$ \\
\hline $1.7,5 \times 10^{-9}$ & $4,516 \times 10^{15}$ & $7,25 \times 10^{-4}$ & $6 \times 10^{-11}$ & 637 & 12500 \\
\hline $2.15 \times 10^{-9}$ & $9,033 \times 10^{15}$ & $1,45 \times 10^{-3}$ & $1,2 \times 10^{-10}$ & 1274 & 25000 \\
\hline $3.3 \times 10^{-8}$ & $1,8066 \times 10^{16}$ & $2,9 \times 10^{-3}$ & $2,4 \times 10^{-10}$ & 2548 & 50000 \\
\hline $4.6 \times 10^{-8}$ & $3,613 \times 10^{16}$ & $5,8 \times 10^{-3}$ & $4,8 \times 10^{-10}$ & 5096 & 100000 \\
\hline $5.12 \times 10^{-8}$ & $7,226 \times 10^{16}$ & $11,6 \times 10^{-3}$ & $9,6 \times 10^{-10}$ & 10190 & 200000 \\
\hline
\end{tabular}


for the given number of ions. Considering that 8 grams of $\mathrm{LiH}$ contains $6.022140857 * 1023$ nucleons [9], Table 2 indicates the energies required and produced by the generator.

\subsection{Main vacuum units of the generator}

The work of the vacuum subsystems of the generator consists of five sequential blockprocesses, which, when completed, give the result in the form of a stable incoming energy from thermonuclear fusion. The processes are controlled by an electronic subsystem. Each vacuum unit has a unique description of the processes that take place in it during operation. Further, the main blocks of lithium hydride evaporator and ionizer-plasma source, streams discretization and formation of the target and other processes are shown schematically in Figures 2-6.

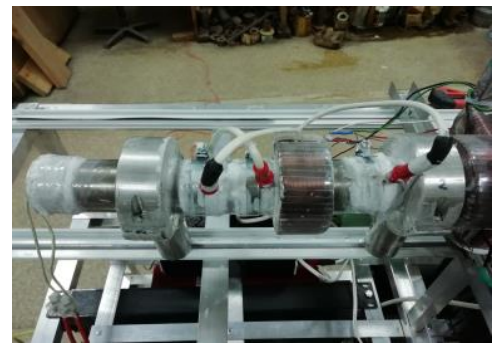

Figure 2. Cartridge and ion plasma source

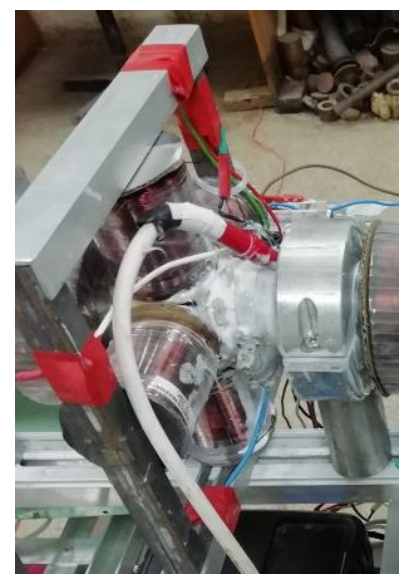

Figure 3. Magneto-optical storage unit in the Figure 4

The QEC-Txx series quantum device is an open cathode HF vacuum triode powered by an external ion source. Compatible with various plasma electric generators and is used as a converter of energy of high-energy ions into high-frequency oscillations.

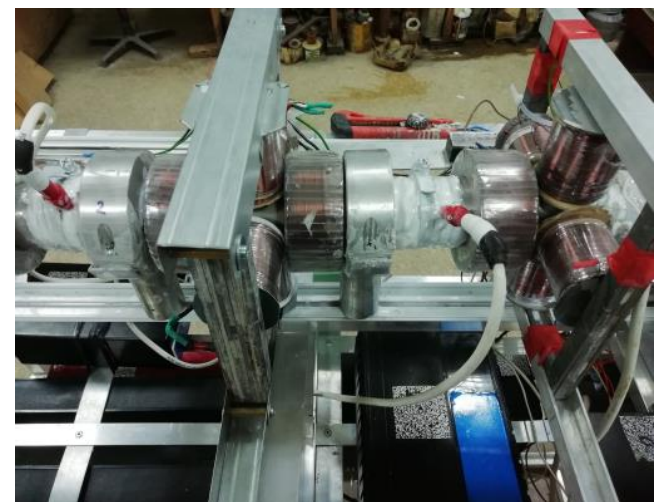

Figure 4. Accelerator the right part of the accelerator

Target bombing, thermonuclear fusion, transition to the second chamber, cycle renewal, see Figs. 5 and 6. For checking the output of the electrical load, see Fig. 7 and data in Tables 3-5 further.

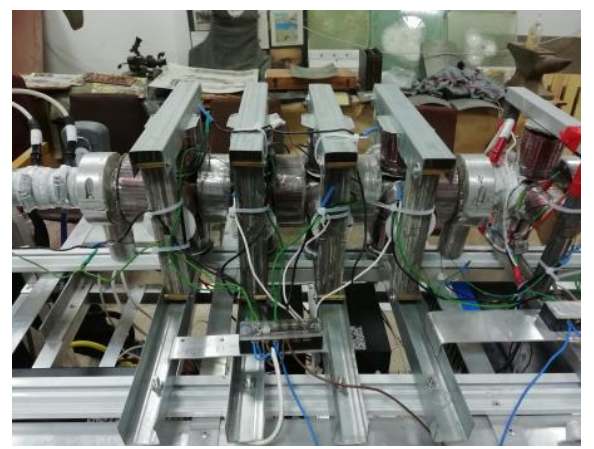

Figure 5. Magneto-optical 4-cycle synthesis chamber following from the properties of quasineutrality and the divergence of the displacement current in this case will be equal to zero, which allows us to exclude one equation of continuity from the system and leave only the ionic one:

Conditions for description of plasma motion in the electromagnetic field in our case, when the plasma passes through the first magneto-optical system into the second, due to an increase in temperature and an increase in density in a gaseous substance, we can use magneto hydrodynamics to describe general processes. Due to the fact that the voltage field and electromagnetic potentials are fixed, we can use the property of "quasineutrality" of plasma flows and consider the problem in a static form, since the parameters of the plasma flows during the transition will not change in any way.

In this case, well known, the number of ions and electrons will be equal;

- Quasineutrality $=n_{i}=n_{e}=n$

- Bias current $=\operatorname{div} \sim j=0$

- Continuity equations $=\frac{\partial n}{\partial t}+\operatorname{div} n v \sim_{i}=0$ 
Thus, in our case, the plasma moves in a magnetostatic field, but which changes discretely. This is a fundamental feature of the electronic control of magnetic fields of the created device for cyclic alternation of magnetoquadrupole and solenoid systems. Calculations of individual magnetic quadrupole lenses for compaction of charged particle fluxes in super-strong magnetic fields, including pulsed ones, and their advantages are well known [11-13].

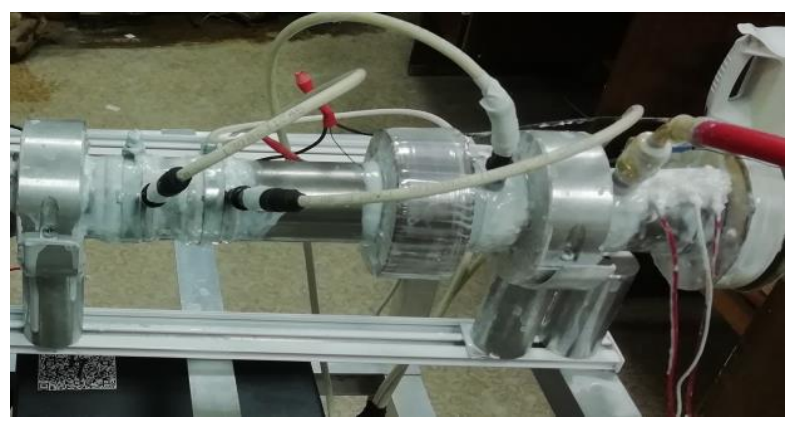

Figure 6. Energy converter, ion vacuum pump and electrical terminal. Transient highfrequency triode - ion emission quantum energy converter (QEC) and ion vacuum pump-Neutralizer.

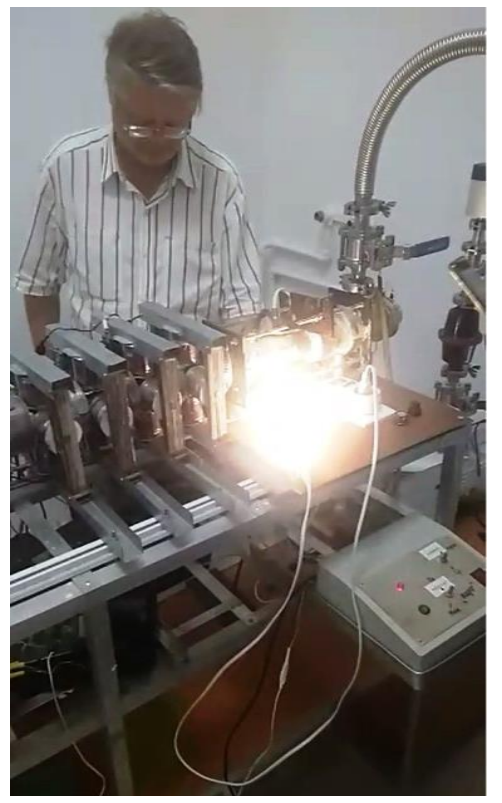

Figure 7. Electrical load output

Description of the motion of the plasma in an electromagnetic field, Ohm's law for an equivalent circuit:

$$
\frac{\vec{j}}{\sigma}=\frac{\nabla p_{e}}{e n}+\left[\vec{E}+\frac{1}{c}\left(\overrightarrow{v_{e}}, \vec{H}\right)\right], \quad \sigma=\frac{\alpha}{e n}
$$

- plasma conductivity, (2) where $\alpha-$ is a coefficient showing the total mass of the particle flow per unit volume.

\subsection{Obtaining controlled discrete flows of charged particles}

The set of methods defining the laws of charged particles parameters changes: energy $\mathbf{E}$, particle current $\mathbf{I}$, concentration $\mathbf{n}$, period Tsl determine the production of electronically controlled flows of charged particles [9, 14]. The approach is based on the formation of the linear flux $\mathbf{P}_{i}$ from ion source with the average value of ion current $\mathbf{I}_{0}$.

The partition of the linear flow $\mathbf{P}_{i}$ with an initial current $\mathbf{I}_{0}$ into flows $\mathbf{I}_{01}, \mathbf{I}_{02}, \ldots, \mathbf{I}_{0 n}$ with the repetition period $\left(\begin{array}{lll}\mathbf{T}_{01} & \ldots & \mathbf{T}_{0 n}\end{array}\right)$ is called current sampling [14], that is $\left(\mathbf{I}_{01}, \mathbf{T}_{01}\right)\left(\mathbf{I}_{02}, \mathbf{T}_{02}\right) \ldots\left(\mathbf{I}_{0 n}, \mathbf{T}_{0 n}\right)$. Thus, we determine $\mathbf{D}_{n I}-$ current discrete.

Let us define for the motion of the discrete D1 the function $\mathbf{F 1}(\mathbf{x}, \mathbf{y}, \mathbf{z}, \mathbf{t})$, which describes its motion in time $\mathbf{t}$ in such a way that the functional discretization takes place $[\mathbf{F 1}(\mathbf{x}, \mathbf{y}, \mathbf{z}, \mathbf{t}) \ldots \mathbf{F n}(\mathbf{x}, \mathbf{y}, \mathbf{z}, \mathbf{t})]$. Thus, we define $\mathbf{D}_{n F}-$ functional discrete. This means practically a sweep for every single stream. The combination of these methods is called the method of compaction of the flow of charged particles and obtaining the primary electronically controlled flow - obtaining discrete flows Dn from the initial linear flow $\mathbf{P}_{i}$.

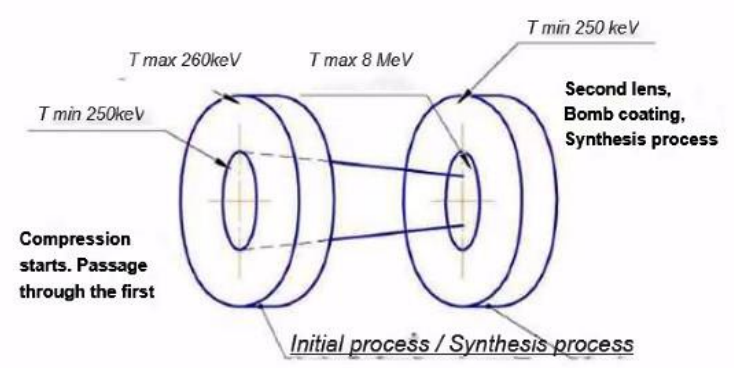

Figure 8. Plasma passage from the first to the second magnetic quadrupole lenses

Plasma passage from the first to the second magnetic quadrupole lens is carried out as follows (Figure 8). First, in the first magnetic lens, the process of compression of the stream of plasma particles takes place, and then the bombardment of a fixed target, defined by the first stream, takes place. The second stream knocks out the targets of the first stream into the second chamber and the process starts over.

Quadrupole lenses consist of two sections rotated by $90^{\circ}$ and containing a winding laid in the grooves of the magnetic circuit with hyperbolic stripes (Figures 9 and 10). 


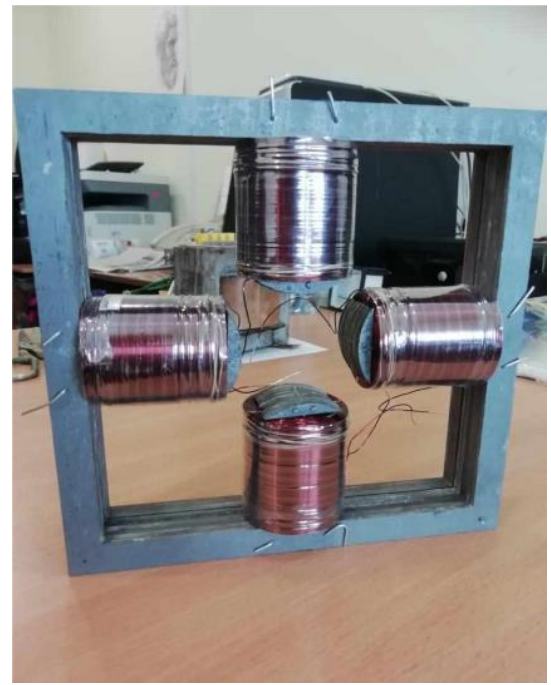

Figure 9. Magnetic quadrupole lens for the magnetooptical drive unit

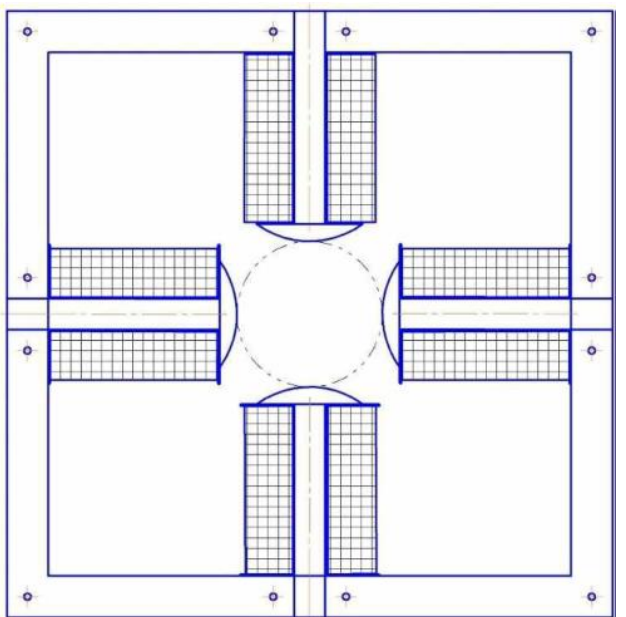

Figure 10. Assembly drawing of a magnetic quadrupole lens

\section{RESULTS AND DISCUSSION 3.1 Distribution of plasma particles character- istics}

The particle velocities are distributed in the following dependence $f(x)=4 \pi \frac{x^{2}}{v^{2}} \exp \left(\frac{-x^{2}}{v^{2}}\right)$, where $v$ flows rates, $x$-the distance from the flow centers. Changes in particle velocities (Fig. 11), participating in thermonuclear fusion, characterize the plasma density, the higher the particle velocity, the higher the plasma density in the fusion chamber. Radial distribution of plasma particles in the accelerating field of the ionic and electronic components, $U=300 \mathrm{kV}$. Plasma in our case, when particle velocities are considering, can also be interpreted as an ideal gas and described by the Boltzmann equation, but the potential energy of the external field can be neglected and the Boltzmann distribution can be translated into the Maxwell distribution:

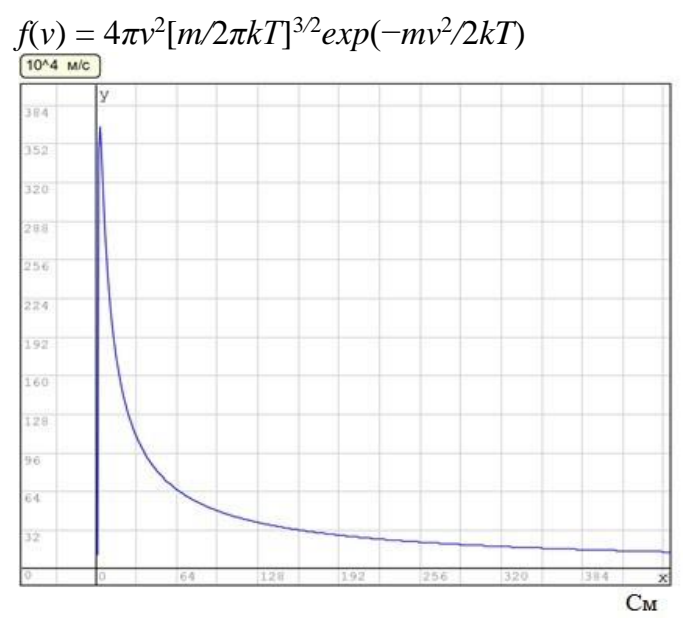

Figure 11. Velocity distribution of plasma particles

The graphs (Figures 11, 12 and 13) show that the main magnetic field is focused in the center of the chamber, where the flow itself is located; the calculation is taken from the origin of coordinates from the very center of the beam.

Distribution of currents (Figure 13) inside the synthesis chamber in our case:

$j(\Delta R)=j_{0}\left(1+\frac{R}{\Delta R}\right)$

where $R$ is the radius of the chamber, $\Delta R$ is the radial displacement, $j_{0}=20 \mathrm{~mA}$ is the initial value of the current.

By changing the parameters of the magnetic lens, it is possible to change the parameters of the currents inside the synthesis chamber, which just affect the distribution of particles in space, which entails a more structured target for more accurate further bombardment with other flows. Current density distribution formula

$\left(j_{1}, j_{2}\right)=\int j_{1}^{*}\left(r^{\prime}\right) j_{2}\left(r^{\prime}\right) d^{3} r^{\prime}$

The distribution of the current density inside the synthesis chamber, where $*$ is the sign of the complex conjugation, $j_{1}$ and $j_{2}$ are the distribution densities of electric or magnetic currents in volume $V$. 


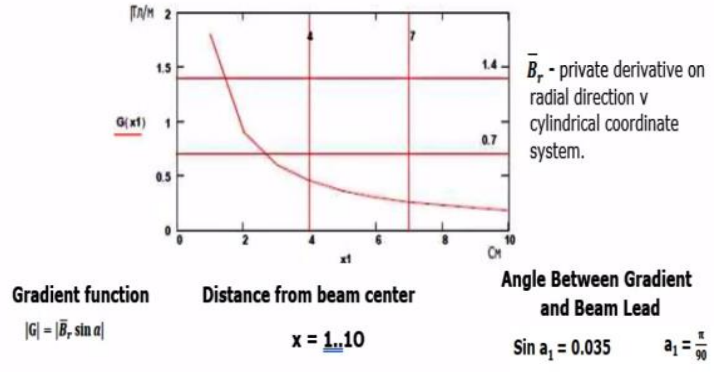

Figure 12. Velocity distribution of plasma particles

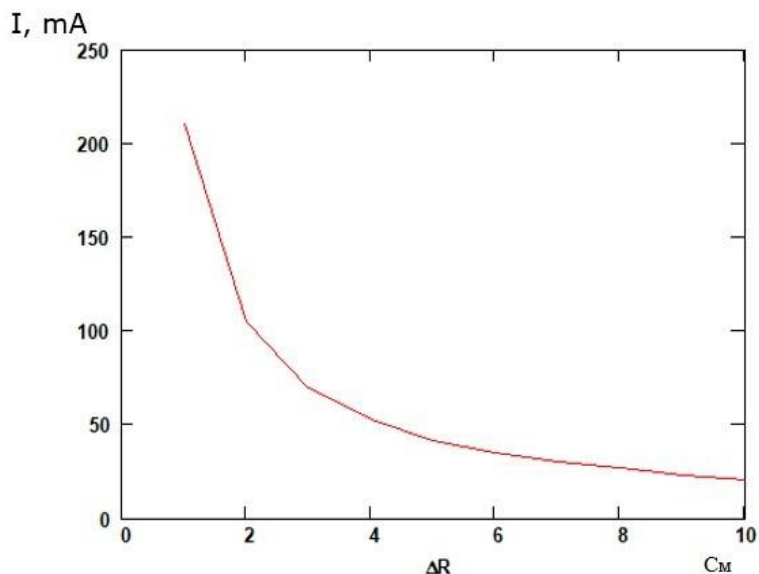

Figure 13. Distribution of currents inside the synthesis chamber

\section{2 installation assembly}

Installation completeness (Figure 14) include:

(i) Vacuum cylindrical cartridge-evaporator with lithium hydride.

(ii) Electronic ion source gun.

(iii) Plasma source of lithium and hydrogen ions.

(iv) Section of the magneto-optical storage device for lithium and hydrogen ions.

(v) Accelerating section of lithium and hydrogen ions.

(vi) Section for the synthesis of lithium and hydrogen ions.

(vii) The quantum power device for a frequency of up to $1 \mathrm{MHz}$.

(viii) Section of the magneto-optical storage of the ion neutralizer chamber.

(ix) Section of the ion neutralizer chamber with an electronic gun. (x) Air cooling system with a heat exchanger (formed at the request of the customer, dependingon the tasks and operating conditions of the installation).

(xi) Electronic generator control system.

(xii) Section of primary high-frequency rectification and filtration.

(xiii) DC-AC electronic unit - converter: $220 \mathrm{~V} / 380 \mathrm{~V}$ (formed at the request of the customer,depending on the installation tasks).

(xiv) Power plant control and protection system.

The base and casing of the generator (formed according to the customer's instructions depending on the installation tasks).

Table 3. Operating parameters of the unit

\begin{tabular}{|c|c|c|c|c|}
\hline \multirow{2}{*}{ Type } & \multicolumn{2}{|c|}{ Stationary PPS - XX V1.0 } & Maximum \\
\cline { 2 - 4 } & $\begin{array}{c}\text { Thermal } \\
\text { rate }\end{array}$ & $\begin{array}{c}\text { Power } \\
\text { rate, } \\
\text { KW }\end{array}$ & $\begin{array}{c}\text { Electric } \\
\text { power, } \\
\text { Kw }\end{array}$ & $\begin{array}{c}\text { power, } \\
\text { kW }\end{array}$ \\
\hline $\begin{array}{c}\text { RPS - } \\
01\end{array}$ & 27 & 20 & 32.5 & 25 \\
$\begin{array}{c}\text { V1.0 S- } \\
020\end{array}$ & & & & \\
\hline
\end{tabular}

\section{3 operating mode of the unit}

PPS - XX V1.0 is a modular system with the generation of electrical energy with a nominal power of 5 $\mathrm{kW}$ of electrical energy. The installation provides the following two modes of operation.

a. "Main power supply mode".

In the main power supply mode, the external line is disconnected from the consumer.

b. "Backup power supply mode".

In the standby power supply mode, the generator is in standby mode and turns on for up to 10 seconds when the external line voltage is lost or insufficient.

Table 4. Parameters of the calculated thermal module

\begin{tabular}{|l|c|}
\hline \multicolumn{2}{|c|}{ Heat exchange module parameters } \\
\hline $\begin{array}{l}\text { Pressure in the regulated heating } \\
\text { outlet, MPa }\end{array}$ & $0,12 \ldots 0,3$ \\
\hline $\begin{array}{l}\text { Hot water temperature in heating } \\
\text { mode, }{ }^{\circ} \mathrm{C}\end{array}$ & 95 \\
\hline $\begin{array}{l}\text { Adjustable heating selection, PPS - } \\
01 \mathrm{~V} 1.0 \text { S-020 nominal (operating } \\
\text { range) consumption, } \mathrm{kg} / \mathrm{h}\end{array}$ & 24 \\
\hline
\end{tabular}


Table 5. AC Power Energy Module

\begin{tabular}{|l|l|l|l|}
\hline \multicolumn{4}{|c|}{ Output voltage of the power energy module AC } \\
\hline$U_{\text {out }}$ & $220 \mathrm{~V}$ & $380 \mathrm{~V}$ & RF output \\
\hline$\omega_{\text {out }}$ & $50-60 \mathrm{~Hz}$ & $50-60 \mathrm{~Hz}$ & $\mathrm{St} 1=250 \mathrm{kHz}$ \\
\hline
\end{tabular}

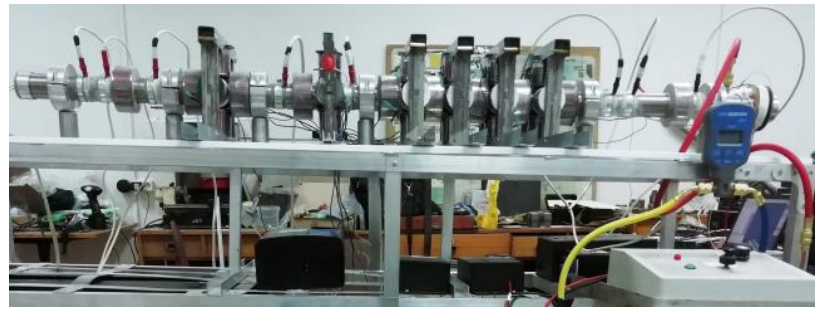

Figure 14. General view of the installation

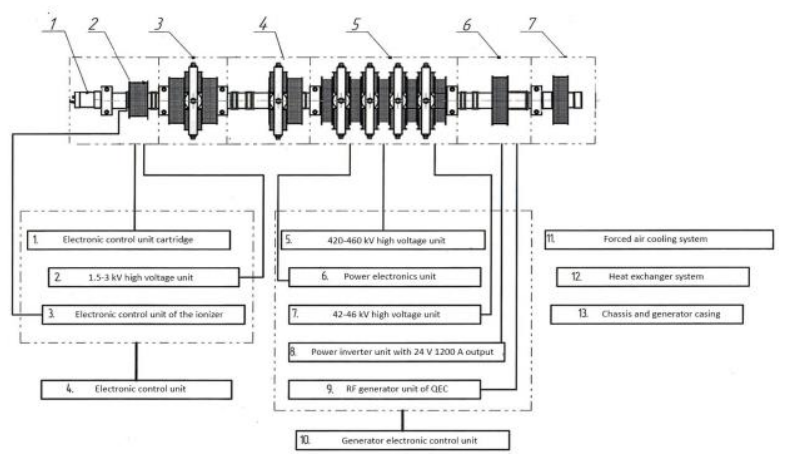

Figure 15. Electronic control scheme of the installation

\section{CONCLUSION}

An electronically controlled plasma electric generator is proposed in considered project, designed to generate energy from the generated controlled ion or plasma flows by specifying a certain law of changing the parameters of magnetic control for the formation of a controlled plasma flow - by discrete change with a given frequency of the direction of magnetic field induction of solenoid magnetic systems and with a pulse-changing value of the magnetic field induction of solenoid systems and magnetoquadrupole lenses and subsequent electronically controlled nuclear fusion in a multi-cycle synthesis chamber and further conversion of the kinetic energy of thermonuclear fusion products into high-frequency pulse-width electric oscillations in a quantum energy converter.

To solve the applied problem of controlled synthesis of light nuclei, a technique and technology for the creation and formation of electronically controlled ion fluxes in a magnetic field by grouping fluxes by discretization and setting certain laws of the sequence of changes in magnetic fields for ion fluxes was developed, an experimental setup with a vacuum subsystem of blocks and an electronic system was assembled control with output characteristics for a constant voltage of $112 \div 24 \mathrm{~V}$, for an alternating voltage of $220 \mathrm{~V}, 50 \div 60 \mathrm{~Hz}$, for a power of 5 to $50 \mathrm{~kW}$, the planned consumption of lithium hydride for operation for three months is $0.75 \mathrm{~kg}$. A power plant with a design capacity of up to $500 \mathrm{~kW}$ has been developed.

Operation of an electronically controlled plasma electric generator PPS - XX V1.0 (Plasma Power System) is based on the synthesis reaction in ion streams obtained as a result of evaporation of lithium hydride in an evaporator cartridge, which is a cylindrical container with a tungsten or tungsten-rhenium heater, and ionization of the resulting vapor in an ionizer, which is a threegrid vacuum device that provides ionization of hydrogen and lithium vapor and their output to a section of a magneto-optical ion storage device, which is a magneto-optical a trap, structurally consisting of a combination of solenoid and quadrupole magnetic systems, and the subsequent acceleration of ions, setting the law of discrete change in the parameters of the magnetic control system and the energy distribution of the generated ion flows with the specified parameters of nuclear fusion, implemented in a pulsed accelerator, which is a span accelerator tube with input and output electrodes, and a magnetic sweep of the flow at the end of the accelerator. The general view of the installation is presented on Fig. 14. Nuclear fusion takes place in a magneto-optical n-cycle synthesis chamber, structurally designed as a combination of various solenoid-quadrupole magneto-optical systems.

The advantages of the device are modularity and compactness of execution, configurability of the system, the possibility of combining individual generators in a synchronously operating monoblock, control of the generator at the hardware and software level.

\section{REFERENCES}

[1] Patent EA 018812, publication date 30.10.2013

[2] Brown J G 2004 The Physics and Technology of Ion Sources (Wiley \& Sons, Incorporated, John) p 396

[3] Mecyach G A 2004 Pulsed power engineering and electronic (Moscow: Nauka) p 704

[4] Forrester A T 1988 Large Ion Beams (New York: Wiley-Interscience) p 325

[5] Akimchenko A et al. 2017 EPJ Web of Conferences 15806004

[6] Radenko V V et al. 2020 Materials of VI-th International Conference LapLas-2020 Part 2 p 159

[7] Gurskaya A V et al. 2020 Materials of International Conference "Fundamental and Applied questions of Physics" 155-163.

[8] Watterson J I W, 2000 International Atomic Energy Agency 31 5-14 
[9] Dolgopolov M V et al., 2019 EPJ Web of Conferences 22202014

[10] Tyrsa V E, Burtseva L P, 2003 Theoretical and Mathematical Physics 48 807-812

[11] Sakharov A D 1995 Scientific works. Collection (Moskow: Publishing House CenterCom) p 528

[12] Sakharov A D 1966 Sov.Phys.Usps 9 294-299

[13] Artsimovich L A, Lukyanov S Yu 1972 Motion of charged particles in electric and magnetic fields (Moscow: Nauka) 224 p.

[14] Radenko A V et al. 2017 Proceedings of conference "Material science. Nonequilibrium phase transformations" 107-108. 